\title{
Finite temperature phase transition with two flavors of improved Wilson fermions
}

\author{
V.G. Bornyakov* \\ Institute for high energy physics, 142281 Protvino, Russia \\ E-mail: vitaly.bornyakoveihep.ru \\ R. Horsley \\ School of Physics and Astronomy, University of Edinburgh, Edinburgh EH9 3JZ, UK
}

\section{Y. Nakamura}

Institut für Theoretische Physik, Universität Regensburg, 93040 Regensburg, Germany

\section{Polikarpov}

Institute of Theoretical and Experimental Physics ITEP, 117259 Moscow, Russia

\section{P. Rakow}

Theoretical Physics Division, Department of Mathematical Sciences, University of Liverpool,

Liverpool L69 3BX, UK

\section{G. Schierholz}

Deutsches Elektronen-Synchrotron DESY, 22603 Hamburg, Germany

\begin{abstract}
The critical temperature is computed for $N_{f}=2$ dynamical flavors of nonperturbatively improved Wilson fermions. The new simulations are performed on lattices $40^{3} x 14$ with lattice spacing and pion mass about $0.08 \mathrm{fm}$ and $200 \mathrm{MeV}$, respectively. We find the deconfinement and chiral phase transitions to coincide within numerical precision. Our results are in broad agreement with a second order phase transition in the chiral limit. The critical temperature at the physical quark mass is found to be $T_{c}=172(3)(6) \mathrm{MeV}$.
\end{abstract}

The XXVIII International Symposium on Lattice Field Theory, Lattice2010

June 14-19, 2010

Villasimius, Italy

\footnotetext{
*Speaker.
} 


\section{Introduction}

The nature of the finite temperature phase transition, and the value of the transition temperature are basic questions in finite temperature QCD which are still missing final answeres. For QCD with $2+1$ flavors one group [1] finds the deconfining transition, and the chiral transition temperatures are separated by $20-30 \mathrm{MeV}$ while another group [2] claims both temperatures to coincide. Moreover, the Brookhaven/Bielefeld collaboration [2] gets for transition temperature $T_{c}=196(3) \mathrm{MeV}$, which is much higher than the transition temperatures found by the Wuppertal group [3] for the deconfining and chiral transitions $-T_{c}=170(7) \mathrm{MeV}$, and $T_{c}=146(5) \mathrm{MeV}$, respectively. Both groups use rooted staggered fermions, but with different levels of improvement. It has been argued [1] that the discrepancy is largely due to the rather coarse lattices used by the Brookhaven/Bielefeld collaboration. Recently, the Brookhaven/Bielefeld collaboration has extended their calculations to lattices of temporal extent $N_{t}=8$ [4] and found that with decreasing lattice spacing $T_{c}$ was shifted by $5-7 \mathrm{MeV}$ towards smaller values.

The connection between deconfining and chiral transition has been subject of several phenomenological considerations. Naively, one would expect the temperature of the deconfinement transition to lie below that of the chiral transition, if different at all. This turns out to be the case, for example, in the Polyakov-loop extended Nambu-Jona-Lasinio model [5]. More likely is that both transitions occur at the same temperature, as Polyakov loop and chiral condensate mix at finite dynamical quark masses. The consequence would be a simultaneous enhancement of both the chiral and Polyakov-loop susceptibilities along the transition line $[6,7,8,9]$.

To clarify the issue, independent investigations of the nature of the finite temperature phase transition preferably with different type of the lattice fermionic action are needed (for recent related works see [11] (domain wall), [12] (twisted mass) and [13] (improved Wilson fermions) ). In this work we present results of simulations with $N_{f}=2$ dynamical flavors of nonperturbatively $O(a)$ improved Wilson fermions and plaquette gauge action on lattices of temporal extent $N_{t}=14,12,10$ and 8. Our results were partially reported in [10, 14].

\section{Definitions and simulation parameters}

The fermionic action for each of the two flavors reads

$$
\begin{aligned}
S_{F}=a^{4} \sum_{x} & \left\{\frac{1}{2 a} \sum_{\mu} \bar{\psi}(x) U_{\mu}(x)\left[\gamma_{\mu}-1\right] \psi(x+a \hat{\mu})\right. \\
& -\frac{1}{2 a} \sum_{\mu} \bar{\psi}(x) U_{\mu}^{\dagger}(x-a \hat{\mu})\left[\gamma_{\mu}+1\right] \psi(x-a \hat{\mu}) \\
& \left.-c_{S W} \frac{i}{2 a} \sum_{\mu \nu} \bar{\psi}(x) \sigma_{\mu \nu} P_{\mu \nu}(x) \psi(x)+\left(m+m_{c}\right) \bar{\psi}(x) \psi(x)\right\}
\end{aligned}
$$

where $P_{\mu v}$ is the clover-leaf form of the lattice field strength tensor and

$$
a m_{c}=\frac{1}{2 \kappa_{c}}, \quad a m=\frac{1}{2 \kappa}-\frac{1}{2 \kappa_{c}}
$$

$\kappa_{c}$ being the critical value of the hopping parameter. 


\begin{tabular}{|c|c|c|l|c|}
\hline$\beta$ & $c_{S W}$ & $V=N_{s}^{3} N_{t}$ & \multicolumn{1}{|c|}{$\kappa_{c}$} & $r_{0} / a$ \\
\hline 5.20 & 2.0171 & $16^{3} 8$ & $0.136050(17)$ & $5.454(58)$ \\
5.20 & 2.0171 & $24^{3} 10$ & $0.136050(17)$ & $5.454(58)$ \\
5.25 & 1.9603 & $16^{3} 8$ & $0.136273(7)$ & $5.880(26)$ \\
5.25 & 1.9603 & $24^{3} 8$ & $0.136273(7)$ & $5.880(26)$ \\
5.25 & 1.9603 & $32^{3} 12$ & $0.136273(7)$ & $5.880(26)$ \\
5.25 & 1.9603 & $40^{3} 14$ & $0.136273(7)$ & $5.880(26)$ \\
5.29 & 1.9192 & $24^{3} 12$ & $0.136440(4)$ & $6.201(25)$ \\
\hline
\end{tabular}

Table 1: Parameters of the simulation.

The couplings, lattice volumes and lattice spacings covered by our simulations are listed in Table 1. The scale parameters $r_{0} / a$ have been taken from the zero temperature runs of the QCDSF collaboration at the corresponding couplings. They refer to the chiral limit $\kappa=\kappa_{c}$. We also list the critical hopping parameters $\kappa_{c}$, which we adopted from QCDSF as well. (For recent relevant work see [15].) The gauge field configurations were generated on the BlueGene/L at KEK, the RSCC cluster at RIKEN, the MVS-100k at the Joint Computer Center (Moscow), on the SKIF-Chebyshev at Moscow State University, as well as on the Altix at HLRN.

Two-flavor QCD is expected to undergo a second order transition at finite temperature in the chiral limit and at very small quark masses. In the chiral limit the order parameter is the chiral condensate

$$
\sigma=\frac{a^{3}}{V} \sum_{x} \bar{\psi}(x) \psi(x)
$$

For heavy quark masses close to the quenched limit, the theory is known to undergo a first order phase transition. In that limit the order parameter is the Polyakov loop

$$
L=\frac{1}{N_{s}^{3}} \sum_{\vec{x}} \operatorname{Re} L(\vec{x}), L(\vec{x})=\frac{1}{3} \operatorname{Tr} \prod_{x_{4}=1}^{N_{t}} U_{4}(x) .
$$

The temperature of the chiral transition is, for general $m$, identified with the peak position of the chiral susceptibility

$$
\chi_{\sigma} \equiv\left\langle\sigma^{2}\right\rangle_{c}=\left\langle\sigma^{2}\right\rangle-\langle\sigma\rangle^{2}
$$

while the peak of the Polyakov-loop susceptibility

$$
\chi_{L} \equiv N_{s}^{3}\left\langle L^{2}\right\rangle_{c},\left\langle L^{2}\right\rangle_{c}=\left(\left\langle L^{2}\right\rangle-\langle L\rangle^{2}\right)
$$

defines the temperature of the deconfining transition.

It is expected that the two-flavor theory is in the same universality class as the three-dimensional $\mathrm{O}(4)$ Heisenberg model [16], with the external magnetic field and the magnetization being identified with the bare quark mass $\hat{m} \equiv a m$ and the chiral condensate $\hat{\sigma} \equiv\langle\sigma\rangle$, respectively. The critical exponents of this model were found to be [17]

$$
\frac{1}{\beta \delta}=0.537(7), \frac{1}{\delta}=0.206(1) .
$$


Let $T_{c}(m)$ denote the pseudocritical temperature at finite $m$, which we define to be the temperature corresponding to the position of the peak of the chiral susceptibility

$$
\chi_{\sigma}=\frac{\partial \hat{\sigma}}{\partial \hat{m}} .
$$

From the scaling relation connecting the chiral condensate, the dynamical quark mass and the temperature in the vicinity of the phase transition we then derive

$$
T_{c}(m)-T_{c}(m=0) \propto \hat{m}^{\frac{1}{\beta \delta}} .
$$

Assuming

$$
m_{\pi}^{2} \propto m,
$$

we thus expect to find

$$
T_{c}(m)-T_{c}(m=0) \propto m_{\pi}^{1.07(1)}
$$

for a second order transition at $m=0$. A first order transition, on the other hand, would give

$$
T_{c}(m)-T_{c}(m=0) \propto m_{\pi}^{2} .
$$

The chiral condensate is related to the average plaquette $P$ by means of a Maxwell relation [18]. The chiral condensate and the plaquette can be found from the partial derivatives of the partition function $Z$ :

$$
\begin{aligned}
& \left.\frac{1}{V} \frac{\partial}{\partial \beta} \ln Z\right|_{\hat{m}}=-6 P+2 \frac{\partial \hat{m}_{c}}{\partial \beta} \hat{\sigma}-2 \frac{\partial c_{S W}}{\partial \beta} \hat{\delta}, \\
& \left.\frac{1}{V} \frac{\partial}{\partial \hat{m}} \ln Z\right|_{\beta}=2 \hat{\sigma},
\end{aligned}
$$

where last term in (2.13) comes from the clover term and is neglected below because it is suppressed by two orders of the lattice spacing with respect to the chiral condensate $[19,20,21]$.

The second derivative $\partial^{2} \ln Z / \partial \beta \partial \hat{m}$ can be expressed in two different orders, which leads to relation:

$$
\left.\frac{\partial P}{\partial \hat{m}}\right|_{\beta}-\left.\frac{1}{3} \frac{\partial \hat{m}_{c}}{\partial \beta} \frac{\partial \hat{\sigma}}{\partial \hat{m}}\right|_{\beta}=\left.\left.\frac{1}{3} \frac{\partial \hat{m}}{\partial \beta}\right|_{\hat{\sigma}} \frac{\partial \hat{\sigma}}{\partial \hat{m}}\right|_{\beta} .
$$

called the Maxwell relation. It holds for any lattice size and for all values of $\beta$ and $m$. Then chiral condensate susceptibility can be expressed as

$$
\chi_{\sigma}=\frac{1}{\mu} \frac{\partial P}{\partial \hat{m}},
$$

where

$$
\mu^{-1}=3\left(\frac{\partial \hat{m}_{c}}{\partial \beta}+\left.\frac{\partial \hat{m}}{\partial \beta}\right|_{\hat{\sigma}}\right)^{-1}
$$

is a finite number.

We also computed the correlator $\langle L \sigma\rangle_{c}$ which can be obtained from the derivative of the average Polyakov loop with respect to mass:

$$
\langle L \sigma\rangle_{c}=\left.\frac{\partial\langle L\rangle}{\partial \hat{m}}\right|_{\beta} .
$$



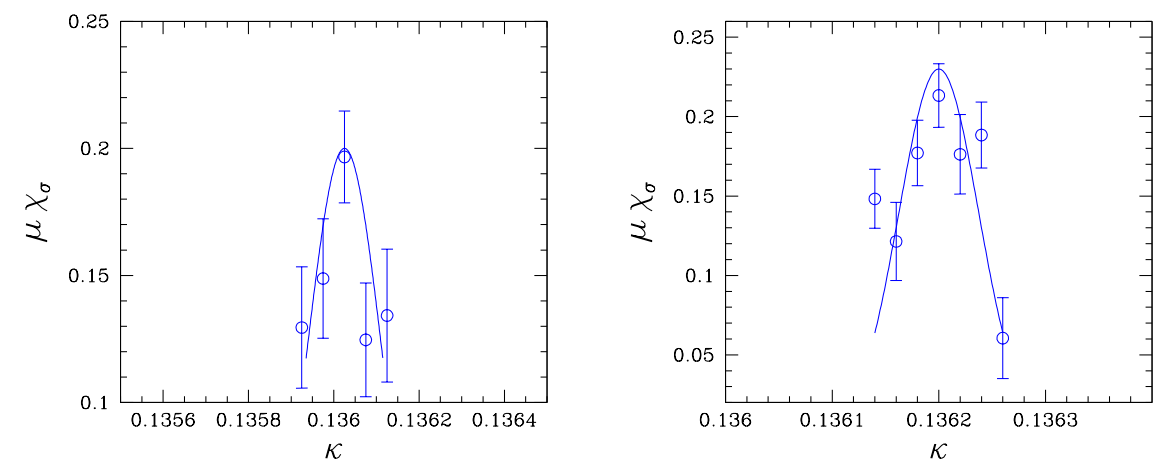

Figure 1: The chiral susceptibility on the $32^{3} 12$ (left) and $40^{3} 14$ lattice (right) at $\beta=5.25$ together with a Gaussian fit.

\begin{tabular}{|c|l|c|l|l|l|}
\hline$\beta$ & \multicolumn{1}{|c|}{$V$} & $r_{0} T_{c}(m)$ & \multicolumn{3}{|c|}{$r_{0} m_{\pi}^{T_{c}}$} \\
& & & \multicolumn{1}{|c|}{$\chi_{L}$} & \multicolumn{1}{|c|}{$\chi_{\sigma}$} & \multicolumn{1}{|c|}{$\langle L \sigma\rangle_{c}$} \\
\hline 5.20 & $16^{3} 8$ & $0.682(7)$ & $2.73(6)$ & $2.78(6)$ & $2.81(7)$ \\
5.20 & $24^{3} 10$ & $0.545(6)$ & $1.59(8)$ & $1.59(16)$ & $1.55(14)$ \\
5.25 & $24^{3} 8$ & $0.735(3)$ & $3.18(4)$ & $3.17(4)$ & $3.33(7)$ \\
5.25 & $32^{3} 12$ & $0.490(2)$ & $1.00(11)$ & $1.05(8)$ & $1.05(7)$ \\
5.25 & $40^{3} 14$ & $0.420(2)$ & & $0.59(6)$ & \\
5.29 & $24^{3} 12$ & $0.517(2)$ & $1.49(8)$ & $1.40(9)$ & $1.3(1)$ \\
\hline
\end{tabular}

Table 2: The pseudocritical temperatures and corresponding pion masses obtained from the peak of $\chi_{L}, \chi_{\sigma}$ and $\langle L \sigma\rangle_{c}$ on our various lattices.

\section{Transition temperature}

In Figure 1 we show the chiral susceptibility for lattices with $N_{t}=12$ and 14 corresponding to our lowest quark masses.

In Table 2 we show the pseudocritical temperature $T_{c}(m)$ and the corresponding pseudocritical pion masses, $m_{\pi}^{T_{c}}$, obtained from the peak of the Polyakov-loop susceptibility, the chiral susceptibility and the correlator (2.17) of $L$ and $\sigma$, respectively. In Fig. 2 the results together with an extrapolation to the chiral limit are presented. We find that on all lattices the individual pion masses $m_{\pi}^{T_{c}}$ coincide with each other within the error bars.

One can see from the Figure 2 that the temperature $T_{c}(m)$ shows an almost linear behavior in the pion mass, in accord with the prediction (2.11) of the $O(4)$ model. We thus may fit the data by the ansatz

$$
T_{c}(m)=C+D\left(r_{0} m_{\pi}\right)^{1.07} .
$$

The result is shown by the solid curve. Setting the scale by the nucleon mass, the QCDSF collabo- 


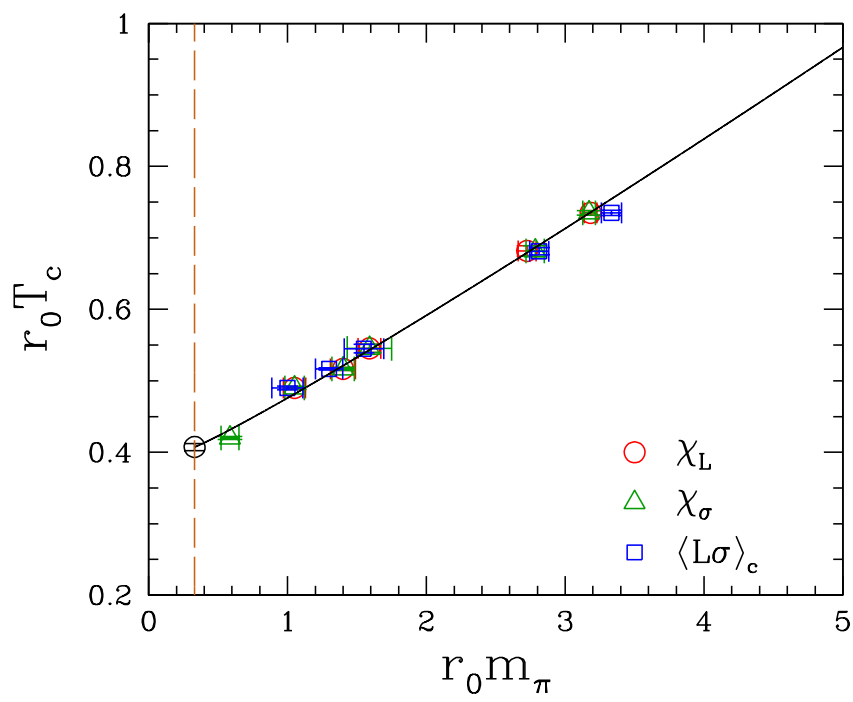

Figure 2: The pseudocritical temperature $T_{c}(m)$ as a function of pion mass, together with a fit to the power $m_{\pi}^{1.07}$, according to the three-dimensional $O(4)$ model.

ration finds $r_{0}=0.467(15) \mathrm{fm}$. Using this value, we obtain at the physical pion mass

$$
r_{0} T_{c}=0.408(5), \quad T_{c}=172(3)(6) \mathrm{MeV},
$$

where the first error on $T_{c}$ is statistical, and the second error reflects the uncertainty in setting the scale. This result only slightly differes from our result [14] obtained without $40^{3} 14$ lattice. It is in good agreement with the deconfining transition temperature found by the Wuppertal group, but lies significantly below the result of the Brookhaven/Bielefeld collaboration.

\section{Conclusions}

We have simulated QCD at finite temperature with two dynamical flavors of nonperturbatively improved Wilson fermions on lattices as large as $N_{t}=14$ and lattice spacings as low as $0.075 \mathrm{fm}$. The transition temperature has been computed from the Polyakov-loop susceptibility, the chiral susceptibility as well as the correlator of Polyakov loop and chiral condensate. All three temperatures are found to coincide with each other within the error bars. The critical behavior appears to be in accord with the predictions of the $O(4)$ Heisenberg model, at least as far as the quark mass dependence of $T_{c}$ is concerned.

Let us note that the Maxwell relation used to compute the chiral condensate susceptibility has proven to be a powerful tool in unveiling the phase structure of clover fermions.

\section{Acknowledgment}

We like to thank the computer centers at KEK (under the Large Scale Simulation Program No. 07-14-B), JSSC RAS (Moscow), Supercomputing Center MSU (Moscow), RIKEN and HLRN (Berlin and Hannover) for their generous allocation of computer time and technical support. 


\section{References}

[1] Y. Aoki, Z. Fodor, S. D. Katz and K. K. Szabo, Phys. Lett. B 643, 46 (2006) [arXiv:hep-lat/0609068].

[2] M. Cheng, N. H. Christ, S. Datta, J. van der Heide, C. Jung, F. Karsch, O. Kaczmarek, E. Laermann, R. D. Mawhinney, C. Miao, P. Petreczky, K. Petrov, C. Schmidt, W. Soeldner and T. Umeda, Phys. Rev. D 77, 014511 (2008) [arXiv:0710.0354 [hep-lat]].

[3] Y. Aoki, S. Borsanyi, S. Dürr, Z. Fodor, S. D. Katz, S. Krieg and K. K. Szabo, JHEP 0906, 088 (2009) [arXiv:0903.4155 [hep-lat]].

[4] A. Bazavov, T. Bhattacharya, M. Cheng, N. H. Christ, C. DeTar, S. Ejiri, S. Gottlieb, R. Gupta, U. M. Heller, K. Huebner, C. Jung, F. Karsch, E. Laermann, L. Levkova, C. Miao, R. D. Mawhinney, P. Petreczky, C. Schmidt, R. A. Soltz, W. Soeldner, R. Sugar, D. Toussaint and P. Vranas, Phys. Rev. D 80, 014504 (2009) [arXiv:0903.4379 [hep-lat]].

[5] S. Roessner, T. Hell, C. Ratti and W. Weise, Nucl. Phys. A 814, 118 (2008) [arXiv:0712.3152 [hep-ph]].

[6] S. Digal, E. Laermann and H. Satz, Eur. Phys. J. C 18, 583 (2001) [arXiv:hep-ph/0007175].

[7] S. Digal, E. Laermann and H. Satz, Nucl. Phys. A 702, 159 (2002).

[8] K. Fukushima, Phys. Lett. B 553, 38 (2003) [arXiv:hep-ph/0209311].

[9] K. Fukushima, Phys. Rev. D 68, 045004 (2003) [arXiv:hep-ph/0303225].

[10] V. G. Bornyakov, S. M. Morozov, Y. Nakamura, M. I. Polikarpov, G. Schierholz and T. Suzuki, PoS LAT2007, 171 (2007) [arXiv:0711.1427 [hep-lat]].

[11] M. Cheng et al., Phys. Rev. D 81 (2010) 054510 [arXiv:0911.3450 [hep-lat]].

[12] F. Burger et al. [ tmfT Collaboration ], PoS LATTICE2010 (2010) 220. [arXiv:1009.3758 [hep-lat]].

[13] B. B. Brandt, H. Wittig, O. Philipsen, L. Zeidlewicz, PoS LATTICE2010 (2010) 172. [arXiv:1008.2143 [hep-lat]].

[14] V. G. Bornyakov et al., Phys. Rev. D 82 (2010) 014504 [arXiv:0910.2392 [hep-lat]].

[15] A. Ali Khan, T. Bakeyev, M. Göckeler, T. R. Hemmert, R. Horsley, A. C. Irving, B. Joó, D. Pleiter, P. E. L. Rakow, G. Schierholz and H. Stüben, Nucl. Phys. B 689, 175 (2004) [arXiv:hep-lat/0312030]; M. Göckeler, R. Horsley, A. C. Irving, D. Pleiter, P. E. L. Rakow, G. Schierholz and H. Stüben, Phys. Lett. B 639, 307 (2006) [arXiv:hep-ph/0409312]; M. Göckeler, R. Horsley, A. C. Irving, D. Pleiter, P. E. L. Rakow, G. Schierholz and H. Stüben, Phys. Rev. D 73, 014513 (2006) [arXiv:hep-ph/0502212]; M. Göckeler, R. Horsley, A. C. Irving, D. Pleiter, P. E. L. Rakow, G. Schierholz, H. Stüben and J. M. Zanotti, Phys. Rev. D 73, 054508 (2006) [arXiv:hep-lat/0601004].

[16] R. D. Pisarski and F. Wilczek, Phys. Rev. D 29, 338 (1984).

[17] K. Kanaya and S. Kaya, Phys. Rev. D 51, 2404 (1995) [arXiv:hep-lat/9409001]; P. Butera and M. Comi, Phys. Rev. B 52, 6185 (1995) [arXiv:hep-lat/9505027]; H. G. Ballesteros, L. A. Fernandez, V. Martin-Mayor and A. Munoz Sudupe, Phys. Lett. B 387, 125 (1996) [arXiv:cond-mat/9606203].

[18] M. Göckeler, R. Horsley, V. Linke, P. E. L. Rakow, G. Schierholz and H. Stüben, Nucl. Phys. B 487, 313 (1997) [arXiv:hep-lat/9605035].

[19] M. Kremer and G. Schierholz, Phys. Lett. B 194, 283 (1987).

[20] T. Doi, N. Ishii, M. Oka and H. Suganuma, Prog. Theor. Phys. Suppl. 151, 161 (2003) [arXiv:hep-lat/0303014].

[21] A. Di Giacomo and Yu. A. Simonov, Phys. Lett. B 595, 368 (2004) [arXiv:hep-ph/0404044]. 